\title{
Clinical Effect of Low Molecular Weight Heparin Sodium Combined with Magnesium Sulfate in the Treatment of Patients with Severe Preeclampsia
}

\author{
Jie Wen'1, Xiuqi Zhang² and Chunji Li²
}

\begin{abstract}
Objective: To investigate the effect of low molecular weight heparin sodium combined with magnesium sulfate in the treatment of patients with severe preeclampsia.

Study Design: An experimental study.

Place and Duration of Study: The Department of Obstetrics and Gynecology, The Affiliated Hospital, North China University of Science and Technology, from April 2016 to July 2017.

Methodology: Patients with severe preeclampsia were randomly divided into observation group (52 cases) and control group (51 cases). In the control group, magnesium sulfate were used, but in observation group low molecular weight heparin sodium were added. After treatment of 7 days, serum TGF- $\beta 1$, VCAM-1, ES levels and endothelial function markers of NO and ET-1 changes were compared between the two groups. The delivery status after treatment was observed in both groups.

Results: After treatment of 7 days, serum TGF- $\beta 1$ and NO levels in the observation group were higher than those in control group (both $p<0.001$ ), and VCAM-1, ES, ET-1, ET-1/NO levels in observation group were lower than those in control group (all $p<0.001$ ). Among the patients who delivered live births, observation group had a longer delivery gestation age after treatment than that of control group $(p=0.038)$; placental quality, body mass and 1 minute Apgar score of surviving newborns in observation group were all higher than those in control group (all $p<0.001$ ).

Conclusion: Low molecular weight heparin sodium combined with magnesium sulfate may improve the prognosis of neonates by reducing the infiltration ability of cytotrophoblasts and regulating endothelial cell function.
\end{abstract}

Key Words: Low molecular weight heparin sodium, Magnesium sulfate, Severe preeclampsia, TGF- $\beta 1$, Endothelial function.

\section{INTRODUCTION}

Severe preeclampsia occurs after 20 weeks of gestation. The clinical signs are mainly hypertension and proteinuria. ${ }^{1}$ The pathogenesis of severe preeclampsia is not yet clear, and it may be related to abnormal cytotrophoblast invasion, vascular endothelial injury, and genetic factors.2,3 The basic pathological changes in severe preeclampsia are systemic arteriole spasm and endothelial dysfunction, which can result in reduced perfusion of heart and blood vessels, liver, kidney, brain and target organs, and related complications.4,5 Severe preeclampsia is one of the major causes of the current maternal and neonatal impairment.

Magnesium sulfate is a common treatment for the prevention of severe preeclampsia from developing

1 Department of Obstetrics and Gynecology, The Affiliated Hospital, North China University of Science and Technology, HeBei Province, 063000, China

2 Department of Obstetrics and Gynecology, Yulin First Hospital, Shaanxi Province, 718000, China

Correspondence: Chunji Li, Department of Obstetrics and Gynecology, Yulin First Hospital, Shaanxi Province, 718000, China

E-mail:mire83615@163.com

Received: May 08, 2018; Accepted: September 27, 2018 into eclampsia. It has the effect of spasmolysis and eclampsia control. 6 However, the effective concentration of magnesium ions in the blood for treatment is very narrow, and high blood magnesium ion concentration will lead to respiratory, liver, and kidney function damage and even sudden death. Therefore, magnesium sulfate cannot be applied for long or in a large quantity. ${ }^{7}$ Low-dose low molecular weight heparin sodium can improve microcirculation and has now been used in the clinical treatment of patients with preeclampsia.8,9 Currently, there is little research on the effect of low molecular weight heparin sodium combined with magnesium sulphate treatment on serum transforming growth factor- $\beta 1$ (TGF- $\beta 1$ ), vascular cell adhesion molecule-1 (VCAM-1), and E-selectin (ES) levels and effects of endothelial function in patients with severe preeclampsia.

In view of this, this study was carried out research to determine the effects of treatment of severe preeclampsia with low molecular weight heparin sodium combined with magnesium sulfate.

\section{METHODOLOGY}

This study was conducted at the Department of Obstetrics and Gynecology, the Affiliated Hospital, North China University of Science and Technology, from April 
2016 to July 2017. Inclusion criteria were patients that met the diagnostic criteria for early-onset severe preeclampsia, patients without serious cardiovascular diseases, liver, kidney, lung or other organ diseases, with singleton pregnancy. Exclusion criteria were patients combined with other diseases during pregnancy such as heart disease, diabetes, serious blood system, endocrine system or immune system diseases, those with diseases that may affect the observation indicators of this study, and patients who were expected to undergo delivery or surgery within 24 hours. The study was reviewed and approved by the Hospital Medical Ethics Committee, and patients and their families signed informed consent. All patients were randomly divided into the observation group (52 cases) and the control group (51 cases).

Both groups of patients were routinely given adequate rest, antihypertensive treatment, moderate oxygen inhalation, dilatation, diuretics, fetal lung maturation, and diet adjustment after admission. At the same time, patients in the control group received low dose intravenous magnesium sulfate. The first dose of intravenous infusion was $20 \mathrm{~mL}$ of $25 \%$ magnesium sulfate mixed with $100 \mathrm{~mL}$ of $5 \%$ dextrose injection completed in one hour. Then intravenous infusion of $60 \mathrm{~mL}$ of $25 \%$ magnesium sulfate mixed with $500 \mathrm{~mL}$ of $5 \%$ dextrose injection was conducted at a rate of 1.5 to $2.0 \mathrm{~g} / \mathrm{h}$. The next day, a dose of $15.0 \mathrm{~g}$ of magnesium sulfate was maintained for intravenous administration. The treatment was continued for 7 days. On the basis of the control group, patients in the observation group were given subcutaneous intraperitoneal injection of low molecular weight heparin at a dose of $5000 \mathrm{U} / \mathrm{time}$, once/day for 7 days, and stopped at 24 hours before termination of pregnancy. The heart rate, blood pressure, fetal heart rate, and 24-hour urine volume during the treatment were closely observed in both groups of patients. Pregnancy was terminated when appropriate. The treatment of spasmolysis, dilatation, diuresis, etc. was given for 1 to 7 days, according to the patient's condition.

After treatment for seven days, $5 \mathrm{~mL}$ morning fasting venous blood was collected from the two groups of patients, and serum was obtained by centrifugation. Serum TGF- $\beta 1$, VCAM-1, ES levels and endothelial function markers ET-1 were determined by enzymelinked immunosorbent assay; NO level was measured using the nitrate reductase method. The delivery status of the two groups of pregnant women were observed and compared, including the number of gestational weeks at the time of delivery, the number of postpartum neonatal deaths, the body mass of surviving newborns, the quality of the placenta, and the $1 \mathrm{~min}$ Apgar score of surviving newborns.

SPSS 23.0 software is adopted for data statistical analysis, measurement data shows in mean $\pm S D$, examined by independent sample t-test. Enumeration data is represented by $\mathrm{n}(\%)$, and X2 test is adopted to examine them. $\mathrm{P}<0.05$ means there is statistical significance in the difference.

\section{RESULTS}

The 103 patients were aged 24-35 years, with the average of $29.53 \pm 3.41$ years, and average gestational age of $34.46 \pm 2.75$ weeks.

After treatment for seven days, serum TGF- $\beta 1$ level was higher in the observation group than that in the control group ( $p<0.001$ ); and VCAM-1 and ES levels were lower than those in the control group ( $p<0.001$ for both), as shown in Table I.

After treatment for seven days, serum NO in the observation group was higher than that in the control group

Table I: Comparison of serum TGF- $\beta 1$, VCAM-1 and ES levels after treatment $7 \mathrm{~d}$.

\begin{tabular}{|c|c|c|c|c|c|c|c|}
\hline \multirow[t]{2}{*}{ Group } & \multirow[t]{2}{*}{$\mathrm{n}$} & \multicolumn{2}{|c|}{ TGF- $\beta 1(p m / L)$} & \multicolumn{2}{|c|}{ VCAM-1 $(\mu \mathrm{g} / \mathrm{L})$} & \multicolumn{2}{|c|}{$\mathrm{ES}(\mathrm{ng} / \mathrm{mL})$} \\
\hline & & Mean \pm SD & p-value & Mean \pm SD & $p$-value & Mean $\pm S D$ & $p$-value \\
\hline Control group & 51 & $184.25 \pm 13.42$ & $<0.001$ & $97.63 \pm 5.81$ & $<0.001$ & $59.85 \pm 7.16$ & $<0.001$ \\
\hline Observation group & 52 & $247.78 \pm 10.18$ & & $46.22 \pm 3.14$ & & $42.14 \pm 5.01$ & \\
\hline
\end{tabular}

Table II: Comparison of serum endothelial function markers after treatment $7 \mathrm{~d}$.

\begin{tabular}{|c|c|c|c|c|c|c|c|}
\hline \multirow[t]{2}{*}{ Group } & \multirow[t]{2}{*}{$\mathrm{n}$} & \multicolumn{2}{|c|}{ ET-1 (ng/L) } & \multicolumn{2}{|c|}{$\mathrm{NO}(\mu \mathrm{mol} / \mathrm{L})$} & \multicolumn{2}{|c|}{ ET-1/NO } \\
\hline & & Mean $\pm S D$ & $p$-value & Mean \pm SD & $\mathrm{p}$-value & Mean \pm SD & $\mathrm{p}$-value \\
\hline Control group & 51 & $115.73 \pm 22.36$ & $<0.001$ & $58.06 \pm 6.35$ & $<0.001$ & $1.97 \pm 0.49$ & $<0.001$ \\
\hline Observation group & 52 & $95.61 \pm 23.58$ & & $71.19 \pm 9.95$ & & $1.32 \pm 0.37$ & \\
\hline
\end{tabular}

Table III: Comparison of delivery after treatment.

\begin{tabular}{|c|c|c|c|c|c|c|c|c|c|}
\hline \multirow[t]{2}{*}{ Group } & \multirow[t]{2}{*}{$\begin{array}{c}\text { Patients who } \\
\text { delivered live births }\end{array}$} & \multicolumn{2}{|c|}{ Delivery gestation age (week) } & \multicolumn{2}{|c|}{ Placental quality (g) } & \multicolumn{2}{|c|}{$\begin{array}{c}\text { Body mass of surviving } \\
\text { newborn }(\mathrm{g})\end{array}$} & \multicolumn{2}{|c|}{$\begin{array}{c}1 \text { min Apgar score of } \\
\text { surviving newborns (score) }\end{array}$} \\
\hline & & Mean \pm SD & $p$-value & Mean \pm SD & $p$-value & Mean $\pm S D$ & $p$-value & Mean $\pm S D$ & $\mathrm{p}$-value \\
\hline Control group & 40 & $33.41 \pm 6.58$ & 0.038 & $301.92 \pm 30.38$ & $<0.001$ & $1976.21 \pm 101.27$ & $<0.001$ & $5.74 \pm 0.90$ & $<0.001$ \\
\hline Observation group & 47 & $36.38 \pm 6.53$ & & $349.76 \pm 22.85$ & & $2403.93 \pm 187.90$ & & $7.83 \pm 0.47$ & \\
\hline
\end{tabular}


$(p<0.001)$; and ET-1 and ET-1/NO were lower than those in the control group (all $p<0.001$ ), as shown in Table II.

Five $(9.62 \%)$ cases of neonatal death occurred in the observation group and $11(21.57 \%)$ cases occurred in the control group. The neonatal mortality rate in the observation group was lower than that in the control group, but there is no statistical difference between them $(p=0.094)$. Among the patients who delivered live births, the observation group had a longer delivery gestation age after treatment than that of the control group $(p=0.038)$; placental quality, body mass and 1-minute Apgar score of surviving newborns in the observation group were all higher than those in the control group (all $p<0.001$ ), as shown in Table III.

\section{DISCUSSION}

Due to the early onset of severe preeclampsia, severe illness, and being accompanied by more complications, its treatment is still one of the more difficult questions for obstetricians. On one hand, in order to improve the neonatal survival rate, it is necessary to prolong the pregnancy time as much as possible. On the other hand, due to the development of the disease, early termination of pregnancy has to be chosen. ${ }^{10,11}$ Therefore, it is particularly important to choose a treatment that can completely improve the hypertension during pregnancy. Now clinical treatment of severe preeclampsia includes antihypertensive drugs, spasmolysis by magnesium sulfate and anticoagulation by low molecular weight heparin. ${ }^{12,13}$ In this study, low molecular weight heparin sodium combined with magnesium sulfate was used to treat severe preeclampsia in pregnant women. There was no statistically significant difference between observation group and control group with respect to the neonatal mortality rate. In the observation group, the postoperative gestational age, placental quality, body mass and 1-minute Apgar score of surviving newborns were better than those in the control group. This suggests that the combination of low molecular weight heparin sodium and magnesium sulfate has a significant effect, and the neonatal prognosis is good.

TGF- $\beta 1$, synthesised by a variety of tissue cells, can regulate cell growth and differentiation. It is an immunosuppressive factor that can inhibit the production of local immune responses. ${ }^{14,15}$ VCAM-1 is a member of the immunoglobulin superfamily and is not expressed in the resting state. It is mainly present in cytokineactivated vascular endothelial cells and mediates the adhesion of lymphocytes and leukocytes to vascular endothelial cells. ${ }^{16,17} \mathrm{E}$-selectin (ES) is only expressed on the surface of endothelial cells activated by cytokines. Under the pathological condition, injured endothelial cells can lead to over-expression of E-selectin, and the secretion of local TGF- $\beta 1$ has an inhibitory effect on the expression of E-selectin. ${ }^{18}$
Studies have shown that TGF- $\beta 1$, VCAM-1 and E-selectin (ES) are involved in the development of preeclampsia. ${ }^{19}$

The results of this study shows that after treatment, serum TGF- $\beta 1$ level is higher in the observation group; than that in the control group and VCAM-1 and ES levels are lower in the observation group than those in the control group. This suggests that the combination therapy of low molecular weight heparin sodium and magnesium sulfate can inhibit the local immune response of the placenta, weaken the infiltration ability of cytotrophoblasts, and reduce vascular endothelial injury.

Previous studies have confirmed severe endothelial dysfunction in patients with severe preeclampsia.20 Vascular endothelial cells can synthesise ET-1 and NO. $\mathrm{NO}$ is synthesised by L-arginine under the action of NO synthase and nicotinamide adenine dinucleotide phosphate. Acting as an endothelium-derived relaxing factor, NO activates the guanylate cyclase in arteries and increases expression of intravascular cyclic guanosine monophosphate, and hence vasodilation. ${ }^{21}$ However, ET-1 can antagonise NO and cause strong contraction of blood vessels. 22 The ratio of ET-1/NO plays an important role in maintaining the dilatation and constriction of the vessels. The results of this study show that after treatment, serum NO level in the observation group is higher than that in the control group, and ET-1 and ET-1/NO are lower than those in the control group. This shows that combination treatment with low molecular weight heparin sodium and magnesium sulfate can more effectively increase NO expression and decrease ET-1 expression and regulate ET-1/NO ratio in patients with severe preeclampsia compared to treatment with magnesium sulfate alone, so that it is even more beneficial in improving the condition of patients with severe preeclampsia.

\section{CONCLUSION}

The effect of low molecular weight heparin sodium combined with magnesium sulfate in the treatment of severe preeclampsia is better than the effect of using magnesium sulfate alone. Low molecular weight heparin sodium combined with magnesium sulfate may improve the prognosis of neonates by reducing the infiltration ability of cytotrophoblasts and regulating endothelial cell function.

\section{REFERENCES}

1. Baghbahadorani FK, Miraj S. The impact of silymarin on improvement of platelet abnormalities in patients with severe preeclampsia. Electron Physician 2016; 8:2436-42.

2. Peng M, Yang M, DingY, Yu L, Deng Y, Lai W, et al. Mechanism of endogenous digitalis-like factor-induced vascular endothelial cell damage in patients with severe preeclampsia. Int $\mathrm{J} \mathrm{Mol}$ Med 2017; 41:985-94.

3. Li X, Li C, Dong X, Gou W. Microrna-155 inhibits migration of trophoblast cells and contributes to the pathogenesis of severe 
preeclampsia by regulating endothelial nitric oxide synthase. Mol Med Rep 2014; 10:550-4.

4. Cozzi V, Garlanda C, Nebuloni M, Maina V, Martinelli A, Calabrese S, et al. PTX3 as a potential endothelial dysfunction biomarker for severity of preeclampsia and IUGR. Placenta 2012; 33:1039-44.

5. Roes EM, Steegers EA, Thomas CM, Geurtsmoespot A, Raijmakers MT, Peters $\mathrm{WH}$, et al. High levels of urinary vascular endothelial growth factor in women with severe preeclampsia. Int J Biol Markers 2004; 19:72-5.

6. Fontenot MT, Lewis DF, Frederick JB, Wang Y, Defranco EA, Groome LJ, et al. A prospective randomized trial of magnesium sulfate in severe preeclampsia: use of diuresis as a clinical parameter to determine the duration of postpartum therapy. Am J Obstet Gynecol 2005; 192:1788-93.

7. Aali BS, Khazaeli P, Ghasemi F. Ionized and total magnesium concentration in patients with severe preeclampsia-eclampsia undergoing magnesium sulfate therapy. $J$ Obstet Gynaecol Res 2008; 34:138-43.

8. Wang S, Hu S, Zhong M. Proteomic investigation of the severe preeclampsia treatment by low molecular weight heparin. Clin Exp Obstet Gynecol 2014; 41:620-6.

9. Sergio F, Maria CD, Gabriella F, Giorgia S, Carolis SD, Giancarlo P, et al. Prophylaxis of recurrent preeclampsia: lowmolecular-weight heparin plus low-dose aspirin versus lowdose aspirin alone. Hypertens Pregnancy 2006; 25:115-27.

10. Quinn KH, Lacoursiere DY, Cui L, Bui J, Parast MM. The unique pathophysiology of early-onset severe preeclampsia: role of decidual t regulatory cells. J Reprod Immunol 2011; 91:76-82.

11. Kuang D, Hua S. Perinatal characteristics and outcomes of the early onset severe preeclampsia. Tianjin Med J 2010; 38:262-5.

12. Gracia VD, Montufar-Rueda C, Ruiz J. Expectant management of severe preeclampsia and preeclampsia superimposed on chronic hypertension between 24 and 34 weeks' gestation. Eur J Obstet Gynecol Reprod Biol 2003; 107:24-7.
13. Anato V, Garmendia JV, Bianco NE, De Sanctis JB. Antihypertensive treatment decreased serum leptin levels in severe preeclampsia during pregnancy. Ann Nutr Metab 2001; 45:190-2.

14. Sinuani I, Averbukh Z, Gitelman I, Rapoport MJ, Sandbank J, Albeck $M$, et al. Mesangial cells initiate compensatory renal tubular hypertrophy via IL-10-induced TGF-beta secretion: effect of the immunomodulator as 101 on this process. Am J Physiol Renal Physiol 2006; 291:384-94.

15. Pulleyn LJ, Newton R, Adcock IM, Barnes PJ. TGF-fbeta1 allele association with asthma severity. Human Genetics 2001; 109:623-7.

16. Mishra A, Guo Y, Li Z, More S, Weng T, Chintagari NR, et al. A critical role for $\mathrm{p} 2 \times 7$ receptor-induced vcam-1 shedding and neutrophil infiltration during acute lung injury. J Immunol 2016; 197:2828-37.

17. Wang L, Li XH, Ning WC. Evaluation of icam-1 and vcam-1 gene polymorphisms in patients with periodontal disease. Med Sci Monit 2016; 22:2386-91.

18. Qiao J, Yang J, Wang Z. Influence of rosuvastatin calcium on cardiac function and se-selectin,TGF- $\beta 1$ in patients with coronary heart disease complicated with paroxysmal atrial fibrillation. Int Med 2017; 12:474-6.

19. Kim SY, Ryu HM, Yang JH, Kim MY, Ahn HK, Lim HJ, et al. Maternal serum levels of vcam-1, icam-1 and e-selectin in preeclampsia. J Korean Med Sci 2004; 19:688-92.

20. Roes EM, Steegers EA, Thomas CM, Geurtsmoespot A, Raijmakers MT, Peters $\mathrm{WH}$, et al. High levels of urinary vascular endothelial growth factor in women with severe preeclampsia. Int J Biol Markers 2004; 19:72-5.

21. Takahashi N, Ishibashi $Y$, Shimada T, Sakane T, Ohata S, Sugamori $\mathrm{T}$, et al. Impaired exercise-induced vasodilatation in chronic atrial fibrillation: role of endothelium-derived nitric oxide. Jap Circul J 2002; 66:583-8.

22. Iglarz M, Clozel M.Mechanisms of ET-1-induced endothelial dysfunction. J Cardiovasc Pharmacol 2007; 50:621-8. 